\title{
Ictus isquémico mesencefálico aislado secundario a ruptura de quiste dermoide
}

\author{
J.M. Ramírez-Moreno; M. Ortega-Martínez*; M.A. Fernández-Gil**; L.M. Bernal-García*; V. Bejarano-Moguel; I.
} Fernández-Portales*; M.J. Gómez-Baquero y J.M. Cabezudo-Artero*

Sección de Neurología. Servicio de Neurocirugía*. Unidad de Resonancia Magnética. Servicio de Radiología**. Complejo Hospitalario Universitario Infanta Cristina. Badajoz.

\section{Resumen}

Los quistes dermoides son lesiones benignas de origen embrionario que representan del 0.04 a $0,25 \%$ de todos los tumores intracraneales. Estos quistes ocasionalmente pueden romperse diseminándose el contenido graso intraquístico al espacio subaracnoideo y/o los ventrículos laterales. En este caso puede provocar diversas manifestaciones clínicas de forma aguda o retardada. El debut de este tipo de tumor con un ictus agudo está escasamente reflejado en la literatura. Presentamos el caso de una mujer de 26 años con un infarto mesencefálico aislado secundario a la ruptura de un quiste dermoide. Discutimos el mecanismo fisiopatológico supuesto y realizamos una revisión de los casos recogidos en la literatura.

PALABRAS CLAVE: Infarto mesencefálico aislado. Ictus. Quiste dermoide. Vasoespasmo. Resonancia magnética cerebral. Tumor cerebral.

Isolated mesencephalic stroke related to a ruptured intracranial dermoid cyst

\section{Summary}

Dermoids cysts are embrionary benign lesions that comprise approximately $0.04-0.25 \%$ of all intracranial tumors. Occasionally they break and spread their content into subarachnoid space and/or lateral ventricles causing several acute or delayed symptoms. Debut of this type of tumor as acute stroke is poorly reflected in literature. We present a 26-year-old woman with a isolated mesencephalic infarct secondary to spontaneous rupture of a dermoid cyst. We discuss the possible pathophysiological mechanisms for this condition and review the literature.

KEY WORDS: Isolated mesencephalic stroke. Dermoid

Recibido: 9-10-08. Aceptado: 29-12-08 cyst. Vasospasm. Magnetic resonance. Stroke. Intracranial tumors.

\section{Introducción}

Los quistes dermoides (QD) son tumores benignos de origen embriológico, de presentación infrecuente; suponen el $0,04-0,6 \%$ de todos los tumores intracraneales. Se localizan generalmente en la línea media de las regiones supraselar, fosa posterior, frontobasal o temporobasal. El quiste está compuesto de una cápsula de epitelio escamoso que contiene grasa, elementos dérmicos, glándulas sebáceas, sudoríparas y folículos pilosos ${ }^{11,20}$. Estas lesiones rara vez puede romperse espontáneamente ${ }^{2}$ o tras un traumatismo craneal $^{16}$, diseminándose su contenido (glóbulos grasos) en el espacio subaracnoideo y los ventrículos laterales. Algunos pacientes tras la ruptura pueden permanecer asintomáticos ${ }^{7}$ y otros presentar diversas manifestaciones clínicas: cefalea, meningitis química, crisis comiciales, confusión, alteraciones visuales, hidrocefalia e ictus establecidos o accidentes isquémicos transitorios ${ }^{10}$.

Los infartos isquémicos mesencefálicos (IIM) habitualmente se encuentran asociados a isquemia de estructuras vecinas (tálamo, protuberancia, cerebelo, córtex temporooccipital), siendo unas 5 veces más infrecuente su presentación aislada. Los IIM aislados (IIMa) en las series suponen del 0,7 al $8 \%$ de todos los ictus de territorio posterior ${ }^{8,12}$.

Presentamos el caso de una mujer de 26 años con un IIMa asociado a un QD roto, discutimos el mecanismo etiopatogénico más probable y realizamos una revisión de los casos recogidos en la literatura.

\section{Caso clínico}

Una mujer de 26 años sin antecedentes neurológicos previos, asmática y en tratamiento con anticonceptivos orales, presenta un cuadro de cefalea holocraneal muy

Abreviaturas. QD: quistes dermoides. IIM: infartos isquémicos mesencefálicos. IIMa: infartos isquémicos mesencefálicos aislados. 


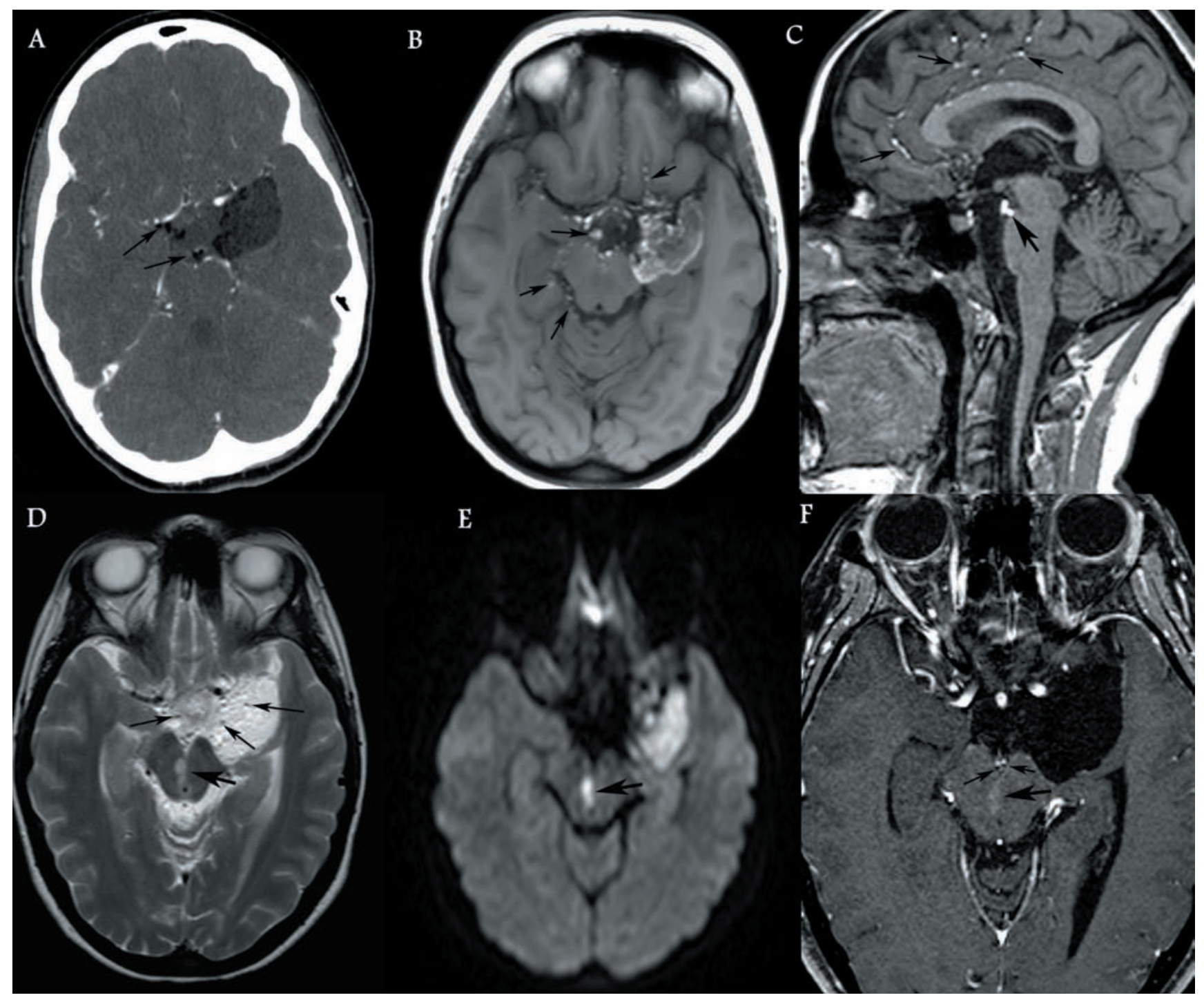

Figura 1. A. TC con contraste: se aprecia una lesión extraaxial adyacente al polo medial del lóbulo temporal izquierdo de baja densidad y pequeñas partículas de bajo valor de atenuación distribuidas por el espacio subaracnoideo de la cisterna supraselar y región interpeduncular (flechas). B. Secuencia TSE potenciada en T1, plano axial: muestra intensidad de señal heterogénea, predominantemente hiperintensa dentro de la lesión y múltiples nódulos brillantes distribuidos por el espacio subaracnoideo (flechas). C. Secuencia TSE potenciada en T1, plano sagital: muestra la diseminación de las partículas por el espacio subaracnoideo (flechas), merecen especial atención las localizadas en el fondo de la cisterna interpeduncular (flecha gruesa). D. Secuencia TSE potenciada en T2, plano axial: la masa muestra alta intensidad de señal y se extiende hacia la cisterna coroidea produciendo efecto de masa sobre el pedúnculo cerebral derecho, en el seno del tumor se aprecian algunas imágenes puntiformes y lineales, hipointensas (flechas) que corresponden al artefacto de desplazamiento químico típico de zonas donde coexisten protones de hidrógeno ligados a agua y a la grasa los cuales procesan a distinta frecuencia; este artefacto sugiere fuertemente la existencia de grasa. Además esta imagen nos muestra una lesión hiperintensa en la región paramedial derecha (flecha gruesa) del tegmento mesencefálico que también brilla en la imagen isotrópica de la secuencia en Difusión (E) (flecha gruesa) siendo sugestiva de lesión isquémica. F. Secuencia eco de gradiente potenciada en T1 con supresión grasa y gadolinio, plano axial: claramente muestra la naturaleza grasa de la masa que cancela su señal, así como la existencia de pequeñas partículas hipointensas adyacentes a los vasos de la cisterna interpeduncular (flechas). El sutil realce de la isquemia mesencefálica subraya la cronología aguda del proceso (flecha gruesa). 
intensa acompañada de fotofobia, nauseas y vómitos sin fiebre de unas 24 horas de evolución, que se complica a la mañana siguiente de forma súbita con inestabilidad para la marcha y diplopia. Acude a urgencias de nuestro centro; en el examen neurológico presenta discreta rigidez nucal, oftalmoplejia internuclear derecha, Romberg tambaleante y lateropulsión izquierda en la marcha, siendo el resto normal. Se le práctica una tomografía axial computarizada objetivándose una lesión con densidad negativa (-20 a -67 Unidades Hounsfield) localizada en la región temporal así como múltiples imágenes ovulares diseminadas por las cisternas supraselares derechas, cuadrigéminas bilaterales, ángulo pontocerebeloso y asta frontal del ventrículo lateral izquierdo que se interpretan como partículas de grasa. Con la sospecha de meningitis química por ruptura de quiste dermoide se inicia tratamiento con dexametasona a dosis de 4 miligramos cada 12 horas. En la resonancia magnética cerebral realizada a los seis días del inicio de los síntomas se observa la masa extraparenquimatosa correspondiente al quiste en la región medial del polo temporal izquierdo que asciende ocupando la zona anterior de la cisterna perimesencefálica izquierda y diseminación de grasa en el espacio subaracnoideo; además se observa en la región paramedial del mesencéfalo una imagen hiperintensa en la secuencias potenciadas en T2 que se muestra hiperintensa en las secuencias en difusión e hipointensa en el mapa de ADC compatible con un infarto isquémico. Se completa la resonancia con secuencias en T1 y EGD con supresión grasa tras administración de gadolinio observándose las pequeñas partículas de grasa diseminadas en el espacio subaracnoideo; llama la atención el acúmulo graso en la cisterna interpeduncular en íntimo contacto con la parte distal de la basilar y la salida teórica de las ramas perforantes mediales mesencefálicas, arterias cerebelosas superiores y arterias cerebrales posteriores. En la figura $1 \mathrm{se}$ muestra una composición con la neuroimagen mas representativa del caso. Tras el resultado de esta prueba se inicia tratamiento con ácido acetilsalicílico a dosis de $100 \mathrm{mg}$ al día y se completó el estudio etiológico del ictus con analítica, hemograma, lipidograma, estudio de autoinmunidad, serología, hormonas tiroideas, homocisteina, anticuerpos anticardiolipina, B2-microglobulina, antifosfolípido, estudio de líquido cefalorraquídeo, electrocardiograma, duplex de troncos supraórticos, doppler transcraneal, test de burbujas, ecocardiografía transtorácica, holter de arritmias $\mathrm{y}$ angioresonancia de polígono de Willis; todas ellas con resultado normal o negativo.

La cefalea respondió a la administración de dexametasona en menos de 48 horas y la oftalmoparesia e inestabilidad se resolvieron a los 10 días quedando la paciente asintomática. Se diagnosticó de infarto aislado anteromedial mesencefálico posiblemente secundario a vasoespamo de arterias perforantes paramedianas mesencefálicas en relación con la diseminación subaracnoidea del contenido graso de un quiste dermoide temporal roto. De acuerdo con la paciente, el servicio de Neurocirugía decidió actitud expectante y controles de neuroimagen seriados, dejando la cirugía electiva para el caso de que apareciera sintomatología por compresión tumoral.

\section{Discusión}

Los QD están compuestos por elementos dérmicos y glándulas sebáceas que producen grasa. La ruptura de un QD es un fenómeno poco frecuente, pero bien documentado en la literatura médica donde hay descritos unos 50 $\operatorname{casos}^{11,20}$. La ruptura generalmente es espontánea ${ }^{2}$, aunque también se ha asociado con traumatismo craneal cerrado ${ }^{16} \mathrm{y}$ en relación con la resección quirúrgica del tumor ${ }^{1}$. Algunos autores han trabajado con la hipótesis de que la ruptura se debe a un crecimiento rápido del QD por la estimulación que los cambios hormonales edad-dependientes inducen en la secreción grasa de las glándulas sebáceas en el interior del quiste ${ }^{20}$. Otros autores están a favor de un mecanismo de tipo mecánico en el que los movimientos cefálicos tendrían un efecto de "martilleo" sobre el QD que provocaría una hendidura y posterior fisura del mismo ${ }^{11}$. El resultado de la ruptura del QD es el esparcimiento de glóbulos grasos por el espacio subaracnoideo y/o el interior de los ventrículos, pudiendo incluso extenderse hacia los surcos cerebrales. Aunque en esta situación el paciente puede quedar asintomático ${ }^{2}$, realizándose el diagnóstico de forma causal a través de un estudio de neuroimagen de rutina, por regla general la ruptura de un QD puede provocar múltiples complicaciones como: meningitis química, cefalea, confusión, crisis comiciales, alteraciones visuales e hidrocefalia ${ }^{10}$.

El caso que presentamos debutó con un síndrome meníngeo y un ictus isquémico aislado anteromedial mesencefálico. La meningitis química es una de las complicaciones mas frecuentes, suele ser transitoria, autolimitada y responder favorablemente a la administración de dexametasona endovenosa ${ }^{1,11}$ tal y como ocurrió en nuestro caso. La isquemia cerebral es otra complicación descrita; en la revisión realizada hemos encontrado 7 casos de isquemia cerebral en relación con la rotura de un QD, en la tabla 1 ofrecemos un resumen de todos ellos $3,4,6,13,14,17,19$. El mecanismo etiopatogénico más probable en estos casos, una vez descartada las causas más frecuentes de ictus en pacientes jóvenes y la compresión de los vasos por la masa tumoral, es el vasoespamo; este es el mecanismo postulado en la mayoría de los casos publicados, en algunos de ellos demostrado angiográficamente ${ }^{4,13}$. La patogenia del vasoespasmo es compleja habiéndose empleado numerosas teorías e implicando a muchas sustancias en la explicación de su génesis; la más defendida es aquella que involucra agentes vasoactivos como causantes. La presencia de 


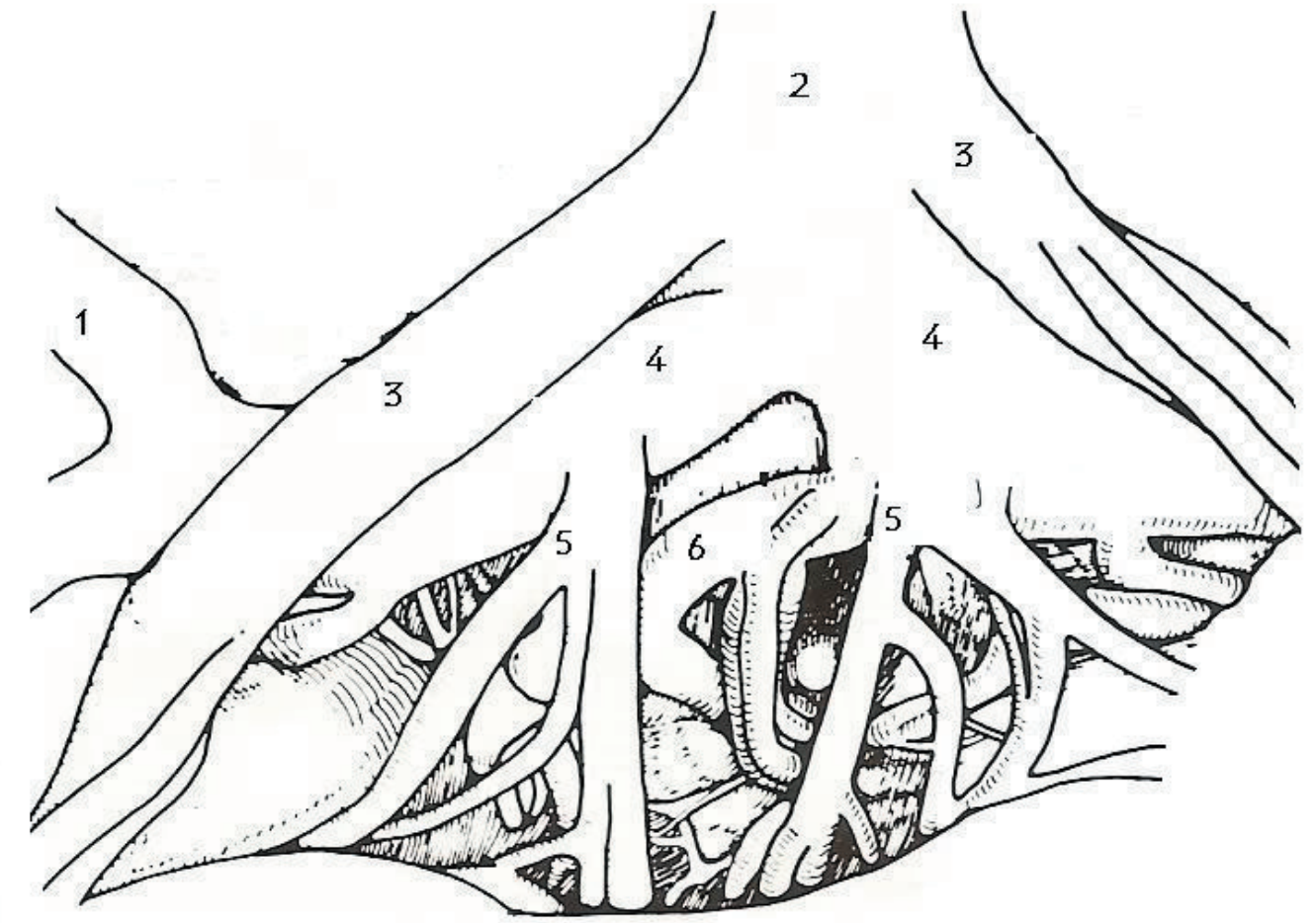

Figura 2. Representación esquemática de la anatomía microvascular de la fosa interpeduncular en una visión anterior. 1. Arteria comunicante posterior, 2. Arteria basilar, 3. Arteria cerebelosa superior, 4. Segmento P1 de la arteria cerebral posterior, 5 Arteria paramediana mesencefálica inferior, 6. Arteria paramediana mesencefálica superior (Cortesía Dr. Cabezudo Artero, modificada de A. Pedroza et al. referencia 15).

lípidos en el espacio subaracnoideo causaría una irritación química en las estructuras neurovasculares que provocaría un espasmo arterial ${ }^{5}$; sin poder descartar como causante el efecto mecánico ejercicio por la partícula grasa en su migración hasta contactar con el vaso. En nuestro caso la relación temporal entre la rotura del QD y el inicio de la sintomatología focal así como la presencia en la cisterna interpeduncular de partículas grasas en íntimo contacto con la salida teórica de las ramas perforantes mediales que vascularizan la región paramedial del meséncefalo hacen plausible este mecanismo etiopatogénico. La anatomía microvascular de esta región es compleja y con gran variabilidad anatómica las arterias mesencefálicas paramedianas inferiores (AMPI) suelen salir de un tronco común de la pared posterior de los últimos cinco milímetros de la arteria basilar, pared posterior o inferior de la arteria cerebelosa superior (ACS) o de la pared inferior del segmento P1 de la arteria cerebral posterior (ACP-P1), mientras que las arterias mesencefálicas paramedianas superiores (AMPS) suelen salir de también un tronco único o común del segmento ACP-P $1^{15}$; en las pruebas de neuroimagen se observa como tanto la punta de la basilar, la salida teórica de la ACS derecha y la ACP izquierda tienen en su proximidad partículas grasas. Está demostrado en modelos animales el efecto irritativo que sobre la arteria basilar tienen los hidroperóxidos lípidos del ácido araquidónico ${ }^{18}$. Se han documentado casos en los que la sintomatología focal no es posible relacionarla temporalmente con la fecha de ruptura del quiste; en esta presentación el mecanismo espástico es mas difícil de considerar; en estos casos es posible que la irritación química provoque una reacción inflamatoria que conduzca a una estenosis focal o difusa de la luz del vaso con aparición de edema en la íntima, necrosis e hiperplasia de la capa muscular con aparición de infiltrado linfocitario y macrófagico tal y como ocurre en la hemorragia subaracnoidea ${ }^{3}$.

La resección quirúrgica del tumor es el tratamiento de elección cuando existe déficit neurológico secundario a la compresión tumoral; en los casos de QD roto generalmente no es posible ni tampoco necesario retirar las partículas grasas esparcidas por el espacio subaraconideo ${ }^{10}$. Se ha comprobado que estas partículas grasas pueden cambiar de lugar y persistir durante años desde el diagnostico sin dar nueva sintomatología $a^{5,9}$. En nuestro caso, de acuerdo con la paciente, optamos por observación clínica y seguimiento con neuroimagen.

El ictus es una complicación que debe ser tenida en cuenta en pacientes con focalidad neurológica asociada a la 


\begin{tabular}{|c|c|c|c|c|c|c|c|c|c|}
\hline \multirow{8}{*}{ 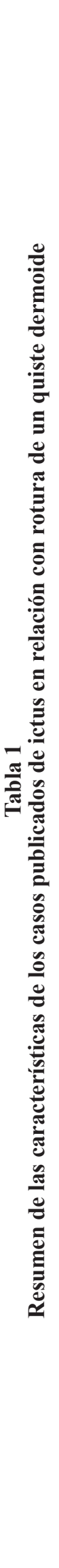 } & 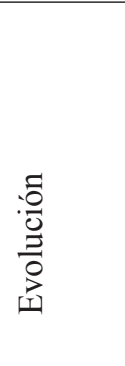 & 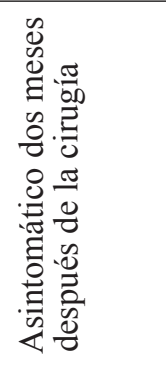 & 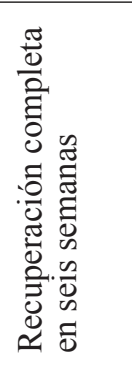 & 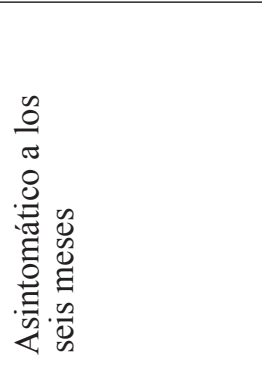 & 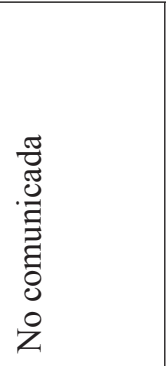 & 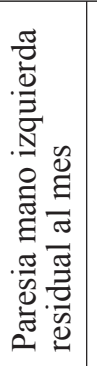 & 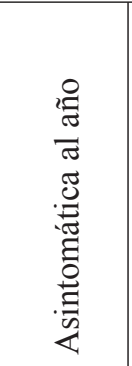 & 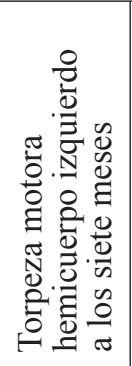 & 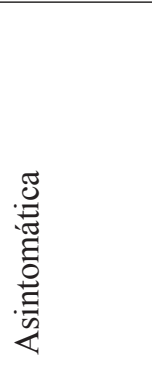 \\
\hline & 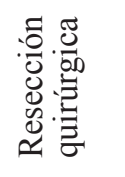 & $\vec{n}$ & $\bar{n}$ & $\bar{n}$ & $\bar{\omega}$ & $\vec{n}$ & $\bar{\sim}$ & $\bar{\infty}$ & $\stackrel{\circ}{z}$ \\
\hline & 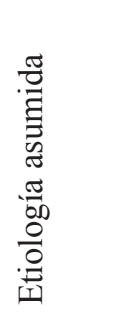 & 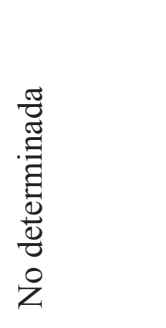 & 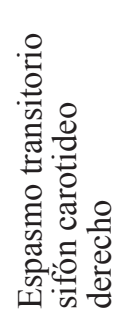 & 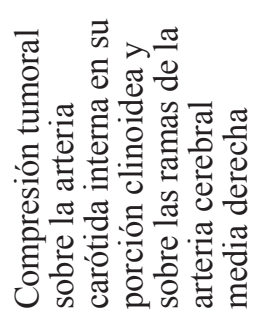 & 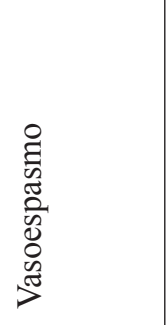 & 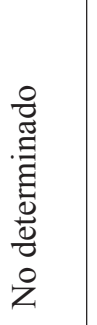 & 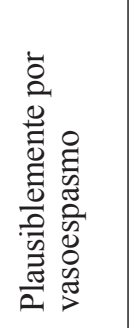 & 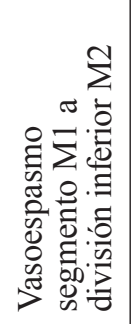 & 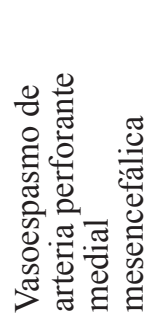 \\
\hline & 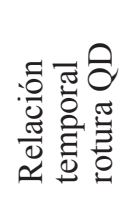 & $\bar{n}$ & $\bar{n}$ & 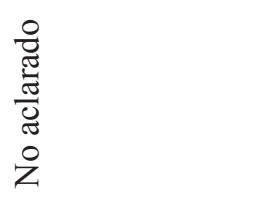 & $\begin{array}{l}\frac{0}{2} \\
\frac{0}{0} \\
0\end{array}$ & 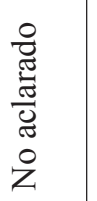 & 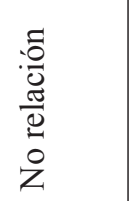 & $\begin{array}{l}0 \\
\frac{0}{0} \\
\frac{0}{0} \\
\frac{\pi}{0} \\
\simeq\end{array}$ & $\bar{n}$ \\
\hline & 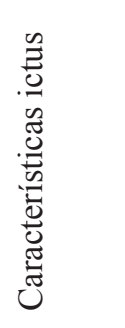 & 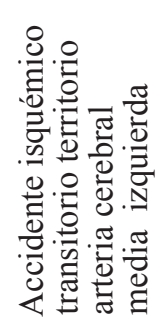 & 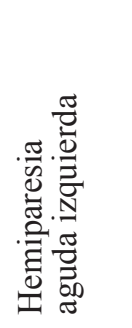 & 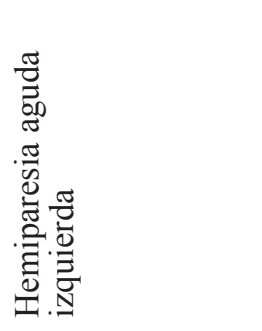 & 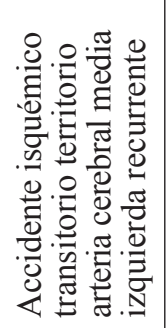 & 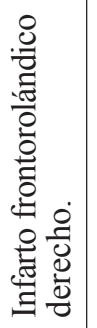 & 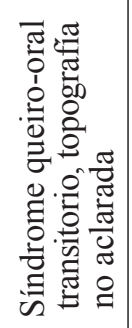 & 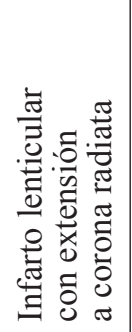 & 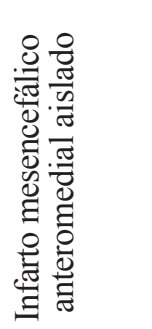 \\
\hline & 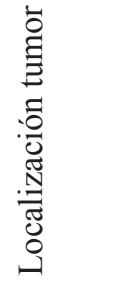 & 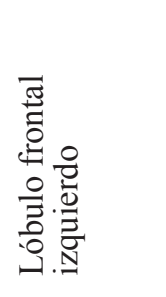 & 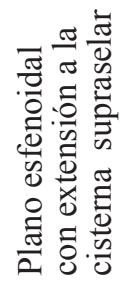 & 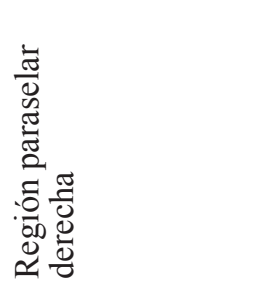 & 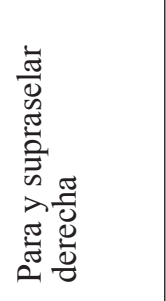 & 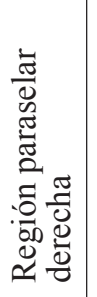 & 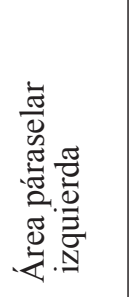 & 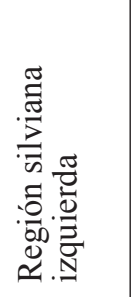 & 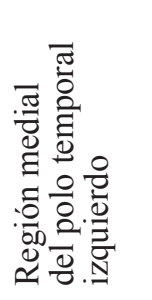 \\
\hline & $\begin{array}{l}\text { च्ञ } \\
\text { 离 } \\
\text { In }\end{array}$ & $\underset{d}{\stackrel{.00}{: ~}}$ & $\underset{n}{\stackrel{0}{0}}$ & $\underset{m}{\grave{0}}$ & $\grave{\curvearrowright}$ & $\underset{\mathrm{o}}{\stackrel{\dot{\vec{\omega}}}{\vec{\Xi}}}$ & 方 $\frac{\overline{\vec{\Xi}}}{\vec{\Xi}}$ & $\grave{\curvearrowright} \cdot \frac{\bar{\Xi}}{\vec{\Xi}}$ & ৩ \\
\hline & 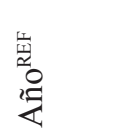 & $\begin{array}{l}\stackrel{\circ}{\infty} \\
\stackrel{2}{=}\end{array}$ & $\begin{array}{l}\stackrel{m}{\sim} \\
\stackrel{\alpha}{=}\end{array}$ & $\stackrel{2}{\partial}$ & $\begin{array}{l}\overline{\hat{n}} \\
\text { }\end{array}$ & $\stackrel{2}{2}$ & $\frac{ \pm}{8}$ & 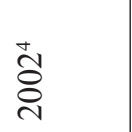 & $\stackrel{\infty}{\stackrel{1}{े}}$ \\
\hline
\end{tabular}


rotura de un quiste dermoide. El mecanismo mas plausible de la isquemia es el vasoespamo, y va a favor de la implicación de agentes vasoactivos en la patogenia del mismo.

\section{Bibliografía}

1. Carvalho, G.A., Cervio, A., Mathies, C., Samii, M.: Subarachnoid fat dissemination after resection of a cerebellopontine angle dysontogenic cyst: case report and review of the literature. Neurosurgery 2000; 47: 760-764.

2. Cerezal, L., Vázquez-Barquero, A., Canga, A., Abascal, F., Bustamante, M., Izquierdo, J.M.: Rotura espontánea de quiste dermoide intracraneal: hallazgos en resonancia magnética. Neurocirugía 1998; 9: 237-240.

3. Civit, T., Pinelli, C., Lescure, J.P., Anxionnant, R., Auque, J., Hepner, H.: Stroke related to a dermoid cyst: case report. Neurosurgery 1997; 41: 1396-1399.

4. Ecker, R.D., Atkinson, J.L., Nichols, D.A.: Delayed ischemic deficit after resection of a large intracranial dermoid: case report and review of the literature. Neurosurgery 2003; 52: 706-710.

5. El-Bahy, K., Kotb, A., Galal, A., El-Hakim, A.: Ruptured intracranial dermoid cyst. Acta Neurochir (Wien) 2006; 148: 457-462.

6. Ford, K., Drayer, B., Osborne, D., Dubois, P.: Case Report: Transient cerebral ischemia as a manifestation of ruptured intracranial dermoid cyst. J Comp Assist Tomogr 1981; 5: 895-897.

7. Funk, M.: Ruptured intracranial dermoid as an incidental finding. Aktuelle Radiol 1995; 5: 232-234.

8. Kumral, E., Bayulkem, G., Akyol, A., Yunten, N., Sirin, H., Sagduyu, A.: Mesencephalic and Associated Posterior Circulation Infarcts. Stroke 2002; 33; 2224-2231.

9. Larsson, E.M., Brandt, L., Holtås, S.: Persisting intraventricular fat-fluid levels following surgery on a ruptured dermoid cyst of the posterior fossa. Acta Radiol 1987; 28: 489-490.

10. Liu, J.K., Gottfried, O.N., Salzman, K.L., Schmidt, R.H., Couldwell, W.T.: Ruptured Intracranial Dermoid Cyst: clinical, radiographic, and surgical features. Neurosurgery 2008; 62: 377-384.

11. Lunardi, P., Missouri, P.: Supratentorial dermoid cyst. J Neurosurg 1991; 75: 262-266.
12.Martin, P.J., Chang, H.M., Wityk, R., Caplan, L.R.: Midbrain infarction: associations and aetiologies in the New England Medical Center Posterior Circulation Registry. J Neurol Neurosurg Psychiatry 1998; 64: 392-395.

13. Mikhael, M.A.: Transient spasm of carotid siphon complicating ruptured cranial dermoid cyst. Radiology 1982; 144: 824 .

14. Nakamura, M., Mizuguchi, M., Momoi, M.Y., Chou, H., Masuzawa, T.: Transient cheiro-oral syndrome due to a ruptured intracranial dermoid cyst. Brain \& Development 2001; 21: 261-263.

15. Pedroza, A., Dujovny, M., Ausman, J.I., Diaz, F.G., Cabezudo Artero, J., Berman, S.K., et al.: Microvascular anatomy of the interpeduncular fossa. J Neurosurg 1986; 64: 484-493.

16. Philips, W.E., Martinez, C.R., Cahill, D.W.: Ruptured intracranial dermoid tumor secondary to closed head trauma. Computed tomography and magnetic resonance imaging. J Neuroimaging 1994; 4: 169-170.

17. Pumar, J.M., Villalón, J.A., Castiñeira, J.A., Vázquez, J.L., Vidal, J.: Ruptura de quiste dermoide paraselar: valoración con TC y RM. Rev Neurol (Barc) 1995; 23: 450-451.

18. Sasaki, T., Wakai, S., Asano, T., Watanabe, T., Kirino, T., Sano, K.: The effect of a lipid hydroperoxide of arachidonic acid on the canine basilar artery. An experimental study on cerebral vasospasm. J Neurosurg 1981; 54: 357-365.

19. Smith, A.S., Benson, J.E., Blaser, S.I., Mizushima, A, Tarr, R.W., Bellon, E.M.: Diagnosis of ruptured intracranial dermoid cyst: value MR over CT. AJNR 1991; 12: 175-180.

20. Stendel, R., Pietila, T.A., Lehmann, K., Suess, O., Brock, M.: Ruptured intracranial dermoid cysts. Surg Neurol 2002; 57: 391-398.

Ramírez-Moreno, J.M.; Ortega-Martínez, M.; FernándezGil, M.A.; Bernal-García, L.M.; Bejarano-Moguel, V.; Fernández-Portales, I.; Gómez-Baquero, M.J.; CabezudoArtero, J.M.: Ictus isquémico mesencefálico aislado secundario a ruptura de quiste dermoide. Neurocirugía 2009; 20: 272-277.

Correspondencia postal: José Ma Ramírez-Moreno. Sección de Neurología. Complejo Hospitalario Universitario Infanta Cristina. Avenida de Elvas s/n. 06080 Badajoz. 